\title{
A régua de carpinteiro (escalas) de Leonard Digges (1520- 1559) para o estudo de conceitos matemáticos: possível incorporação na Educação Básica
}

Resumo: A utilização de documentos históricos, como recursos didáticos advindos da História da Matemática, possibilita construções de interfaces entre a história e o ensino. Dentre esses documentos, encontra-se o tratado A Booke Named Tectonicon, publicado no ano de 1556, pelo inglês Leonard Digges (1520-1559), que apresenta três instrumentos de medida. A partir disso, este artigo tem o intuito de apresentar conceitos matemáticos incorporados nas escalas graduadas da régua de carpinteiro. Para isso, fez-se necessário realizar uma pesquisa qualitativa de cunho documental, efetuando uma tradução da obra original, visando compreender os conhecimentos matemáticos presentes no tratado. Após observar o lado do instrumento que apresenta as escalas graduadas, nota-se, em sua construção, a mobilização de conceitos matemáticos como unidades de medida e frações. Assim, conclui-se que o processo de construção e graduação da parte da frente da régua de carpinteiro possui itens potencialmente didáticos, voltados, principalmente, para a incorporação de instrumentos matemáticos ao ensino.
Sabrina de Sousa Paulino

Aluna da Licenciatura em

Matemática pela Universidade

Estadual do Ceará (UECE). Ceará,

Brasil.

iD orcid.org/0000-0002-8309-8757

$\triangle$ sabrina.paulino@aluno.uece.br

Ana Carolina Costa

Pereira

Doutora em Educação. Professora do Programa de Pós-Graduação em

Educação da Universidade Estadual do Ceará (UECE). Ceará, Brasil

(iD) orcid.org/0000-0002-3819-2381

$\triangle$ carolina.pereira@uece.br

Recebido em 05/01/2021

Aceito em 16/03/2021

Publicado em 08/04/2021

Palavras-chave: Régua de Carpinteiro. Unidade de Medidas. Ensino de Matemática.

\section{Leonard Digges (1520-1559) carpenter's ruler (scales) for the study of mathematical concepts: possible incorporation in Basic Education}

Abstract: The use of historical documents as didactic resources from the history of mathematics, makes it possible to build interfaces between history and teaching. Among these documents, the treatise A Booke Named Tectonicon, published in the year 1556, by the Englishman Leonard Digges (1520-1559), which presents three measuring instruments. Thus, this article aims to present mathematical concepts incorporated in the graduated scales of the carpenter's ruler. For this, a qualitative research of documentary nature was carried out, carrying out a translation of the original work, specific to the mathematical knowledge present in the treatise. After observing the side of the instrument that presents as graduated scales, note in its construction the mobilization of mathematical concepts such as units of measure and fractions. Thus, it is concluded that the construction and graduation process of the front part of the carpenter's ruler, has potentially didactic items, mainly aimed at incorporating mathematical instruments into teaching.

Keywords: Carpenters Ruler. Units of Measure. Mathematics Teaching. 


\title{
Leonard Digges (1520-1559) regla de carpintero (escalas) para el estudio de conceptos matemáticos: posible incorporación en la Educación Básica
}

Resumen: El uso de documentos históricos como recursos didácticos de la historia de las matemáticas, permite construir interfaces entre la historia y la enseñanza. Entre estos documentos, el tratado A Booke Named Tectonicon, publicado en el año 1556, por el inglés Leonard Digges (1520-1559), que presenta tres instrumentos de medida. Así, este artículo tiene como objetivo presentar conceptos matemáticos incorporados en las escalas graduadas de la regla de carpintero. Para ello, se realizó una investigación cualitativa de carácter documental, realizando una traducción de la obra original, con el objetivo de comprender los conocimientos matemáticos presentes en el tratado. Luego de observar el lado del instrumento que presenta las escalas graduadas, se nota en su construcción la movilización de conceptos matemáticos como unidades de medida y fracciones. Así, se concluye que el proceso de construcción y graduación de la parte frontal de la regla de carpintero, tiene elementos potencialmente didácticos, principalmente enfocados a la incorporación de instrumentos matemáticos en la enseñanza.

Palabras clave: Regla de Carpintero. Unidades de Medida. Enseñanza de las Matemáticas.

\section{Introdução}

Durante os séculos XV a XVII, a Europa passou por um grande processo de expansão territorial e, com isso, fez-se cada vez mais necessário que se obtivessem novos conhecimentos, não só matemáticos, mas astronômicos, marítimos etc. Segundo AlfonsoGoldfarb (1994, p. 16),

\begin{abstract}
foi no mundo europeu, cercado por todos os lados, onde começou a fermentar as sementes da Ciência Moderna. Ninguém conseguiu até hoje provar com certeza se essas sementes da Ciência foram o que ajudou os europeus a arrebentarem seus muros e se expandirem por todo o planeta. Ou se, ao contrário, por terem começado a arrebentar os muros, eles puderam trazer, de outras partes para a Europa, as ideias (...) com que regaram e fizeram brotar essas sementes.
\end{abstract}

Assim, muitos praticantes das Matemáticas ${ }^{1}$ iniciaram, durante os séculos XVI e XVII, um intensivo processo de produção de tratados, com o foco principalmente voltado para as matemáticas práticas, incorporando a utilização de instrumentos às áreas de navegação, agrimensura e artilharia.

Dentre esses tratados, destaca-se, para este estudo, o A Booke Named Tectonicon,

\footnotetext{
${ }^{1}$ Saito (2015, p. 172) define os praticantes das matemáticas como sendo um "grupo de estudiosos ingleses que se dedicavam às matemáticas práticas, fabricando instrumentos e escrevendo tratados".
} 
escrito pelo inglês, estudioso das Matemáticas, Leonard Digges (1520 - 1559), publicado, pela primeira vez, em Londres, no ano de $1556^{2}$; “com uma linguagem matemática não tão explícita, o tratado proporciona, ao artesão agrimensor, métodos de resolver problemas de ordem prática presentes em seu cotidiano" (PAULINO, ARGEMIRO FILHO e PEREIRA, 2020, p. 6).

Além de apresentar instrumentos de medida e sua forma de manipulação, Digges (1605) também expõe, em seu tratado, tabelas de medidas, cuja finalidade é auxiliar o processo de graduação e manipulação desses instrumentos. Com isso, este estudo tem o objetivo de identificar e apresentar os conceitos matemáticos incorporados na construção física do instrumento e no processo de graduação das escalas incorporadas a ele, com ênfase nas relações entre suas unidades de medidas.

Para isso, o artigo é dividido em três partes. Inicialmente, é realizada uma breve análise contextual a respeito do tratado A Booke Named Tectonicon. Posteriormente, são expostos os passos realizados no caminho metodológico deste estudo. Em seguida, é apresentada a construção física da régua de carpinteiro e o processo de graduação das escalas da régua. Por fim, é feita uma discussão sobre a possível inserção do instrumento régua de carpinteiro no ensino de Matemática.

\section{Metodologia}

Para que o objetivo do estudo fosse alcançado, inicialmente, fez-se necessário efetuar uma tradução e leitura do tratado original, intitulado A Booke Named Tectonicon, publicado, pela primeira vez, no ano de 1556, pelo inglês, praticante das Matemáticas, Leonard Digges. Dessa forma, como o documento primário utilizado é um texto do século XVI, realizou-se o que Kripka, Scheller e Bonotto (2015) descrevem como uma pesquisa qualitativa de cunho documental, que ocorre quando

os dados obtidos são estritamente provenientes de documentos, com o objetivo de extrair informações neles contidas, a fim de compreender um fenômeno; é um procedimento que se utiliza de métodos e técnicas para a apreensão, compreensão e análise de documentos dos mais variados tipos; é caracterizada como documental quando essa for a única abordagem qualitativa, sendo usada como método autônomo (p. 244).

\footnotetext{
${ }^{2}$ Porém, para esta pesquisa, utiliza-se a edição publicada no ano de 1605.
} 
Além disso, foi realizada, concomitantemente, uma pesquisa bibliográfica em fontes secundárias (CASTILLO, 2016; GILLIPSIE, 1971), o que “é indispensável nos estudos históricos. Em muitas situações, não há outra maneira de conhecer os fatos do passado senão com base em dados secundários" (GIL, 2008, p. 69), com o foco principalmente voltado para se conhecer as circunstâncias sociais, econômicas e políticas em que autor e obra estão inseridos.

\section{O tratado}

Escrito pelo inglês Leonard Digges (1520-1559), o A Booke Named Tectonicon (Figura 1), teve sua primeira edição publicada no ano de 1556, em Londres. Segundo Saito (2015, p. 172), Tectonicon tem o objetivo de "oferecer ao leitor técnicas e procedimentos para resolver diferentes problemas matemáticos". De fato, conforme Digges (1605), seu tratado tem o objetivo de apresentar formas rápidas e precisas de realizar cálculos e obter a medida de áreas.

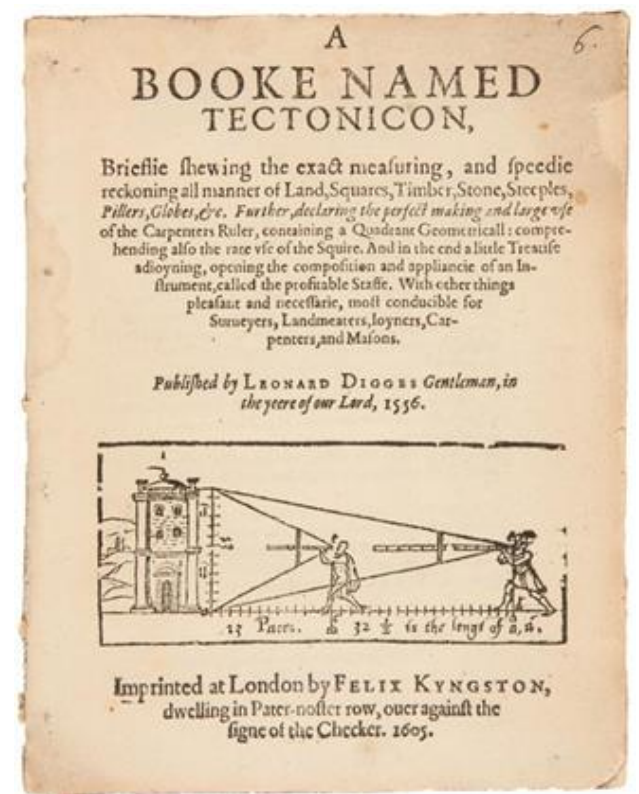

Figura 1: A Booke Named Tectonicon 1605 (DIGGES, 1605, frontispício)

O tratado é dividido em duas seções, cuja primeira delas apresenta 21 capítulos, contendo conhecimentos matemáticos básicos, como os caracteres numéricos, frações, círculos e triângulos. Além disso, a primeira seção também expõe dois instrumentos de medida, a régua de carpinteiro (carpenters ruler) e o esquadro de carpinteiro (carpenters square). A segunda seção traz outros quatro capítulos destinados à construção e 
manipulação de um instrumento chamado báculo (cross-staffe).

Como forma de proporcionar um apoio à construção e manipulação desses instrumentos, Digges (1605) também dispõe algumas tabelas (Quadro 1), que apresentam valores referentes às medidas utilizadas naquele período.

Quadro 1: As tabelas de Digges (1605) e seus conteúdos

\begin{tabular}{|c|c|}
\hline Nome da tabela & Medidas apresentadas \\
\hline Tabela de dados & Valores sem unidades expostas. \\
\hline Tabela de quadrados & Polegadas quadradas, polegadas e partes de polegadas. \\
\hline $\begin{array}{c}\text { Tabela de medidas da } \\
\text { madeira }\end{array}$ & $\begin{array}{r}\text { Polegadas quadradas, pés quadrados, pés, polegadas e partes } \\
\text { de polegadas. }\end{array}$ \\
\hline Tabela de conversões & Pés, polegadas e partes de polegadas. \\
\hline
\end{tabular}

Fonte: Elaboração das Autoras

Ele nomeia essas tabelas de: tabela de dados, tabela de raízes, tabela de medidas da madeira e tabela de conversões. Nelas, estão dispostas unidades de medidas, como as polegadas quadradas, pés e polegadas, como mostra o Quadro 1. Dessa forma, para este estudo, destacam-se o instrumento régua de carpinteiro e a tabela de medidas de madeira, cujos valores têm grande participação no processo de graduação do instrumento, uma vez que, segundo Digges (1605, XII, p. 1), “o efeito dessa régua está acima declarado por tabelas"3.

\section{A construção física da régua de carpinteiro}

A régua de carpinteiro, primeiro dos três instrumentos de medida apresentados em Tectonicon, é composta por duas faces: a primeira contém duas escalas graduadas e a segunda apresenta um quadrante geométrico. Digges (1605, XII, p. 1) explica que a régua é "um instrumento também conhecido e comum"4 Portanto, apesar de expor informações a respeito da construção física do instrumento, ele não se detém em dispor muitos detalhamentos sobre esse processo.

\footnotetext{
${ }^{3}$ Em inglês, lê-se: "the effect of this ruler is above declared by tables" (DIGGES, 1605, XII, p. 1).

${ }^{4}$ Em inglês, lê-se: "an instrument also well known and commune among good artificers" (DIGGES, 1605, XII, p. 1).
} 
Ele ainda ressalta que, para realizar essa construção, deve-se observar as figuras apresentadas no tratado, que têm o objetivo de facilitar a compreensão do leitor quanto a esse processo e, a partir disso, "cobrir sua régua, ambos com madeira e medidas bordadas"5 (DIGGES, 1605, XII, p. 1).

Posteriormente, Digges (1605) discorre sobre as unidades de medida utilizadas para realizar a construção da régua e sobre as dimensões do instrumento, ou seja, sua largura, espessura e comprimento, sendo

abcd bem plana, doze polegadas de comprimento, de um quarto de polegada de espessura, e duas polegadas de largura. Verdadeiramente ela seria mais cômoda, se tivesse dois pés de comprimento esta régua aqui imaginei somente com um pé de comprimento [...] (DIGGES, 1605, XII, p. 1) .

Com base nisso, notam-se algumas características relacionadas à régua. A primeira delas é que o "abcd", ao qual o autor se refere, trata-se dos vértices do instrumento, que ele indica, no texto, utilizando letras minúsculas ${ }^{7}$, porém, na figura representada no tratado, os vértices são representados com letras maiúsculas, como podese observar na Figura 2. Vê-se, aqui também, que Digges (1605) utiliza as polegadas ${ }^{8}$ como unidades de medida para construir seu instrumento. Além disso, o autor explica que a régua deve possuir 12 polegadas de comprimento, 2 polegadas de largura e um quarto de polegada de espessura.

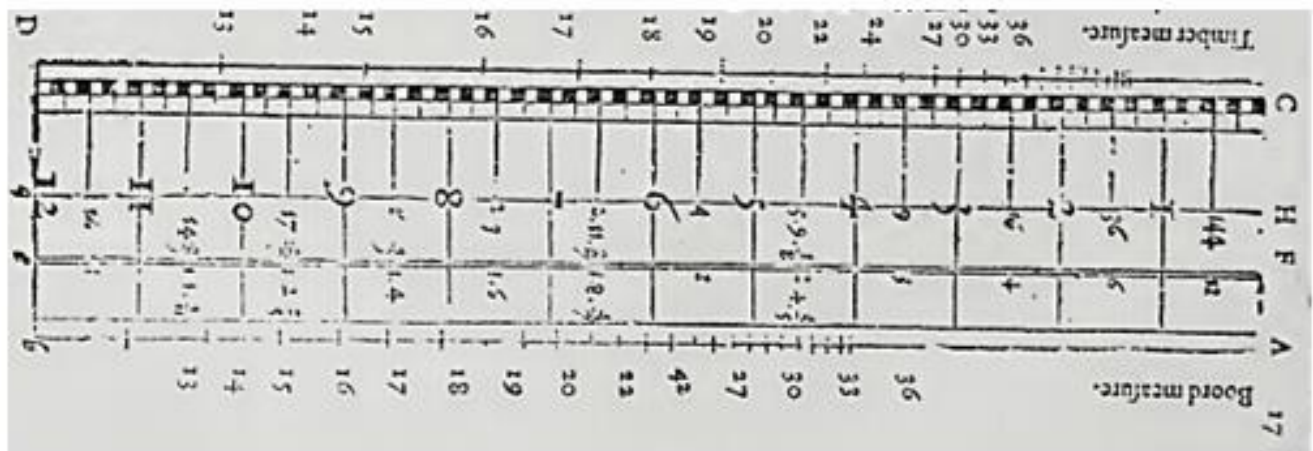

Figura 2: Parte da frente da régua de carpinteiro (DIGGES, 1605, XII, p. 1)

\footnotetext{
${ }^{5}$ Em inglês, lê-se: "to back your ruler, both with timber and board measure" (DIGGES, 1605, XII, p. 1).

${ }^{6}$ Em inglês, lê-se: "a b c d well planned, twelve inches long, a quarter of an inch thick, and two inches in breadth. Truly it were more commodious, if it had two foot in length. This ruler here imagined but a foot in lenght" (DIGGES, 1605, XII, p. 1).

${ }^{7}$ Para uma melhor compreensão, neste estudo, utilizaremos uma linguagem matemática atual.

${ }^{8}$ A polegada é uma medida do sistema imperial, seu uso foi muito comum na Inglaterra do século XVI e sua medida foi tida, inicialmente, como a distância entre a base da unha e a ponta do dedo.
} 
Digges (1605) ainda ressalta que a régua seria mais cômoda se fosse construída com 2 pés ${ }^{9}$ de comprimento, porém, apesar da comodidade ser maior, ele construiu o instrumento somente com 1 pé de comprimento. Com isso, pode-se observar que 1 pé de medida equivale, de acordo com as dimensões apresentadas pelo autor, a 12 polegadas e, portanto, as medidas utilizadas por ele, no século XVI, assemelham-se às utilizadas atualmente para pés e polegadas.

A partir disso, pode-se apresentar, em centímetros, as dimensões dispostas, inicialmente, pelo autor em polegadas (Tabela 1), visto que, atualmente, utiliza-se 2,54 $\mathrm{cm}$ para representar o valor de uma polegada.

Tabela 1: Conversão das dimensões da régua de polegadas para centímetros

\begin{tabular}{c|c|c}
\hline Dimensões da Régua & Polegadas & Centímetros \\
\hline Comprimento & 12 & $30,48 \mathrm{~cm}$ \\
\hline Largura & 2 & $5,08 \mathrm{~cm}$ \\
\hline Espessura & $1 / 4$ & $0,63 \mathrm{~cm}$ \\
\hline
\end{tabular}

Fonte: Elaboração das Autoras

Observando as dimensões da régua descritas por Digges (1605) e as medidas convertidas na unidade de medida moderna ${ }^{10}$, percebe-se que ela é de um tamanho próximo à uma régua escolar, que tem, em média, 30 centímetros de comprimento. Dessa forma, seu manuseio e deslocamento, para locais de seu uso, podem ser realizados com muita praticidade.

\section{O processo de graduação das escalas da régua}

Após apresentar os comentários introdutórios a respeito da construção física do instrumento, Digges (1605) aborda instruções mais específicas sobre a graduação das escalas, localizadas na parte da frente da régua. É importante ressaltar que o autor descreve duas escalas graduadas (Figura 3), em que a primeira mostra medidas de pés e polegadas e a segunda está relacionada às medidas de partes de polegadas e polegadas

\footnotetext{
${ }^{9}$ Medida pertencente ao sistema imperial; seu uso é muito comum na Inglaterra desde o século XVI até os dias de hoje. O valor atual utilizado para representar 1 pé é 12 polegadas ou 30,48 $\mathrm{cm}$.

${ }^{10}$ Medidas pertencentes ao Sistema Internacional de Unidades.
} 
quadradas.

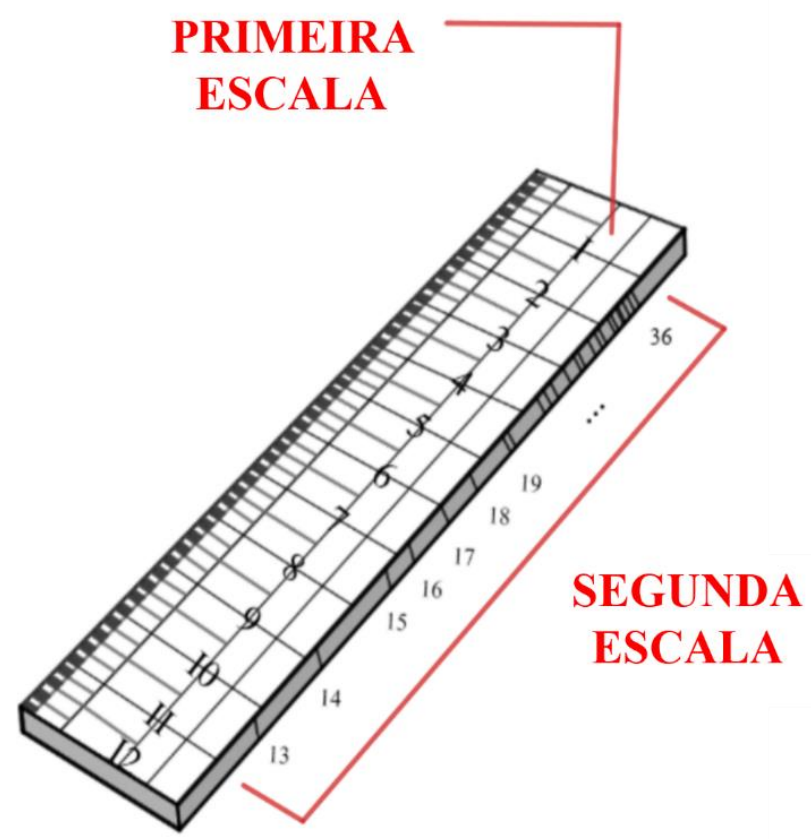

Figura 3: Esquema gráfico da régua de carpinteiro (Elaboração das Autoras)

No que se refere à primeira escala, o autor descreve que ela

está dividida primeiro em doze partes iguais, chamadas polegadas: então cada polegada ao meio, ou duas porções iguais: cada metade em dois quartos: cada quarto em quatro ou duas partes: como neste exemplo. Em seguida, estão os números colocados de 1 a 12 manifestando as polegadas (DIGGES, 1605, XII, p. 1$)^{11}$.

Sabendo, de acordo com as instruções apresentadas por Digges (1605), que a régua possui exatamente 12 polegadas de comprimento, pode-se dividi-la inicialmente em 12 partes iguais (parte 1). Cada parte, posteriormente, será dividia ao meio, em porções de igual medida, gerando, assim, 24 partes (parte 2). O próximo passo é dividir tais porções em duas partes iguais, gerando 48 partes (parte 3) e, por fim, dividir essas partes em duas porções iguais, cada uma gerando 96 partes (parte 4) (Figura 4).

\footnotetext{
${ }^{11}$ Em inglês, lê-se: "is divided first in twelve even parts, called inches: then every inch in half, or two equal portions: each half in two quarters: every quarter in four or two parts at the left: as in this example. Then are the figures placed fro 1 to 12 manifesting the inches" (DIGGES, 1605, XII, p. 1).
} 


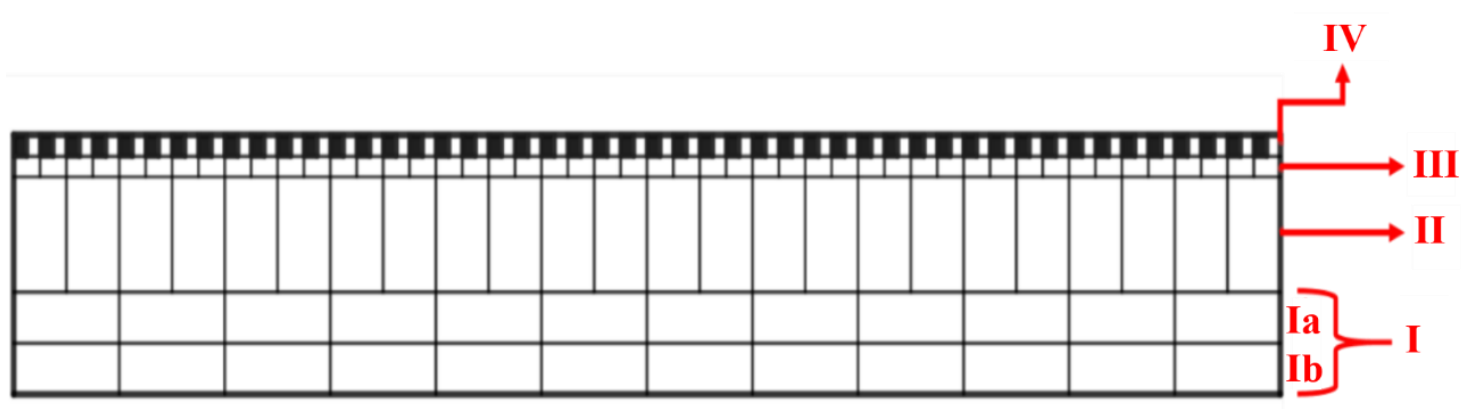

Figura 4: Representação gráfica da primeira escala (Elaboração das Autoras)

A partir da conversão feita anteriormente, compreende-se que 12 polegadas equivalem a 30,48 cm e, com isso, pode-se descobrir o valor de cada divisão realizada por Digges (1605) na construção da primeira escala. Para obtê-lo, basta dividir 30,48 cm (valor total do comprimento da régua) pelo número de partes a serem divididas (Tabela 2).

Tabela 2: As divisões da primeira escala

\begin{tabular}{l|c|c|c|c}
\hline Divisões realizadas & 12 partes & 24 partes & 48 partes & 96 partes \\
\hline Medidas de cada parte & $2,54 \mathrm{~cm}$ & $1,27 \mathrm{~cm}$ & $0,635 \mathrm{~cm}$ & $0,3175 \mathrm{~cm}$ \\
\hline
\end{tabular}

Fonte: Elaboração das Autoras

Consequentemente, Digges (1605, XII, p. 1) ressalta que "sua régua está pronta para receber as medidas que estão marcadas ou figuradas em sua régua"12. Apesar de explicar que a régua está pronta, o autor não menciona nada a respeito da construção da segunda escala, o que nos remete a pensar que ela será apresentada posteriormente.

As medidas, que o autor indica estarem marcadas na régua, pertencem a uma tabela situada no capítulo X, intitulada tabela de medida da madeira (Figura 5), na qual estão expostos valores referentes às medidas de polegadas quadradas, pés, polegadas e partes de polegadas.

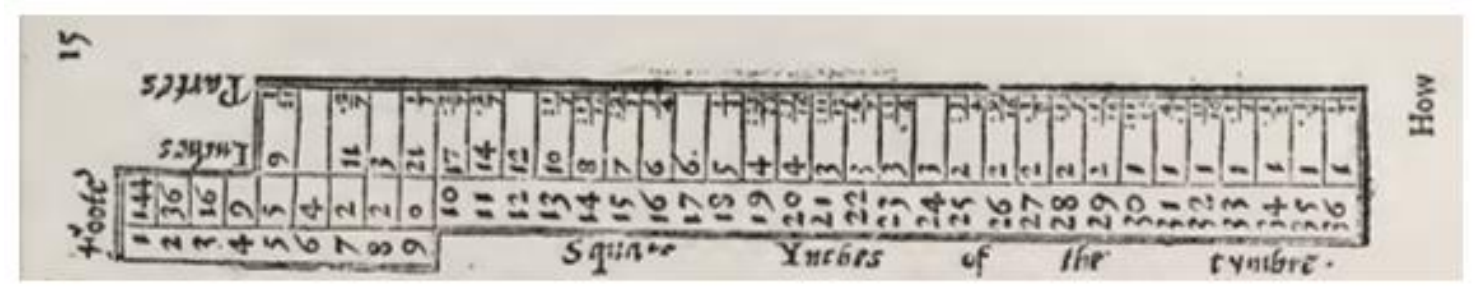

Figura 5: Tabela de medida da madeira (DIGGE, 1605, X, p.1)

\footnotetext{
${ }^{12}$ Em inglês, lê-se: "Thus your ruler is ready to receave the measures which are marked or figured on your ruler thus" (DIGGES, 1605, XII, p. 1).
} 
Digges (1605, XII, p. 2) explica que, para graduar a primeira escala, deve-se "recorrer à sua tabela de medida de madeira, e buscar quantos pés pertencem a 1 polegada quadrada: achareis 144. Este número anote, escreva, ou melhor, grave" ${ }^{\text {"13 }}$.

De acordo com o autor, basta seguir esse processo 12 vezes, que correspondem às 12 polegadas gravadas na régua e a primeira escala estará graduada. Porém, observando as instruções do autor e os valores expostos na primeira escala da régua ${ }^{14}$, notou-se que os valores da tabela de medidas da madeira estão representados somente na parte $1 \mathrm{~b}$ da escala (Tabela 3).

Tabela 3: As 12 medidas da parte $1 \mathrm{~b}$ da primeira escala

\begin{tabular}{c|c}
\hline Polegadas & Medidas da parte 1b \\
\hline 1 & 144 \\
\hline 2 & 36 \\
\hline 3 & 16 \\
\hline 4 & 9 \\
\hline 5 & $59 \frac{3}{25}$ \\
\hline 6 & 4 \\
\hline 7 & $211 \frac{2}{7}$ \\
\hline 8 & 23 \\
\hline 9 & $19 \frac{1}{3}$ \\
\hline 10 & $15 \frac{2}{7}$ \\
\hline 11 & $12 \frac{2}{7}$ \\
\hline 12 & 12 \\
\hline
\end{tabular}

Fonte: Elaboração das Autoras

\footnotetext{
${ }^{13} \mathrm{Em}$ inglês, lê-se: "resort to your table of timber measure, and seek how many feet belong to linch square: there ye shall find 144. This number note, write, or rather grave" (DIGGES, 1605, XII, p.2).

${ }^{14} \mathrm{O}$ artigo proposto é derivado de uma pesquisa envolvendo história da Matemática, que preserva características do documento original, visto que a vertente historiográfica escolhida é atualizada. Assim, decidiu-se manter a notação original do documento A Booke Named Tectonicon, 1605, de Digges (15201559).
} 
Note que, além da parte $1 \mathrm{~b}$, a única outra parte da primeira escala que recebe a incorporação de valores é a parte 1a (Figura 4). É importante salientar que Digges (1605) não dispõe, em capítulos anteriores ou posteriores, informações a respeito do processo realizado para obter tais valores.

Entretanto, observando essas medidas, notou-se uma relação entre elas e o resultado obtido por meio do processo de divisão de 12 pelas polegadas enumeradas de 1 a 12 na escala $\left(\right.$ Pol. $\left.{ }^{15}\right)$. Iniciando pela primeira polegada, tem-se o seguinte processo de divisão: $\frac{12}{1}=12$.

A partir disso, obtém-se o primeiro valor disposto na parte 1a da primeira escala, 12. Consequentemente, esse processo é realizado para obter os 4 primeiros valores representados na escala (Tabela 4$)$.

Tabela 4: Os quatro primeiros valores dispostos na parte 1a da primeira escala

\begin{tabular}{c|c|c}
\hline Pol. & 12/Pol & Polegadas \\
\hline 1 & $\frac{12}{1}$ & 12 \\
\hline 2 & $\frac{12}{2}$ & 6 \\
\hline 3 & $\frac{12}{3}$ & 4 \\
\hline 4 & $\frac{12}{4}$ & 3 \\
\hline
\end{tabular}

Fonte: Elaboração das Autoras

Contudo, a partir da quinta divisão, os resultados não são apenas valores inteiros e os números fracionários começam a aparecer, como é destacado na Figura 6.

Para obter tal resultado, novamente, será necessário realizar o processo de divisão de 12 pela polegada referente à quinta linha, no caso 5, que pode ser reescrita da seguinte forma:

$$
\frac{12}{5}=\frac{10}{5}+\frac{2}{5} \Rightarrow \frac{12}{5}=2+\frac{2}{5}
$$

\footnotetext{
${ }^{15}$ Notação utilizada para representar a unidade de medida polegada.
} 


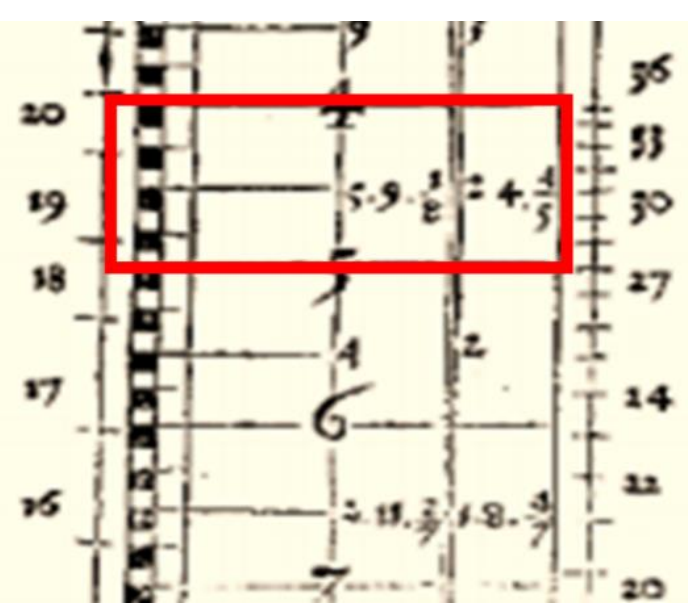

Figura 6: A quinta linha da primeira escala (DIGGES, 1605, XII, p. 1, destaque nosso)

Portanto, obtém-se o resultado quanto à quinta linha da escala de 2 polegadas e 2/5 partes de polegadas. É importante evidenciar que, além de valores fracionários, Digges (1605) apresenta, nas oitava e nona linhas, valores denotados com vírgula, conhecidos, na escrita moderna, como decimais. Para obter todas as 12 medidas da escala, repete-se o processo anterior para todas as 12 polegadas da primeira escala, como é exposto na Tabela 5 .

Tabela 5: Valores dispostos na parte 1a da primeira escala

\begin{tabular}{c|c|c}
\hline Pol. & $\mathbf{1 2 / \text { Pol }}$ & Medidas parte 1a \\
\hline 1 & $\frac{12}{1}$ & 12 \\
\hline 2 & $\frac{12}{2}$ & 6 \\
\hline 3 & $\frac{12}{3}$ & 4 \\
\hline 4 & $\frac{12}{4}$ & 3 \\
\hline 5 & $\frac{12}{5}$ & $2 \frac{2}{5}$ \\
\hline 6 & $\frac{12}{6}$ & 2 \\
\hline 7 & $\frac{12}{7}$ & $1+\frac{5}{7}$ \\
\hline 8 & $\frac{12}{8}$ & 1,5 \\
\hline
\end{tabular}




\begin{tabular}{c|c|c}
\hline 9 & $\frac{12}{9}$ & 1,4 \\
\hline 10 & $\frac{12}{10}$ & $1+\frac{2}{10}$ \\
\hline 11 & $\frac{12}{11}$ & $1+\frac{1}{12}$ \\
\hline 12 & $\frac{12}{12}$ & 1 \\
\hline
\end{tabular}

Fonte: Elaboração das Autoras

Logo, está finalizada a graduação da primeira escala. Por fim, ele apresenta instruções de como devemos graduar a segunda escala da régua. Segundo Digges (1605, XII, p. 2), devemos procurar o valor correspondente a 13 polegadas quadradas na tabela de medida da madeira, que resultará em " 10 polegadas e $1 / 5$. Isto deve ser numerado na linha c d denotando a espessura da régua"16.

A partir dessas informações, notam-se algumas características a respeito da segunda escala do instrumento. Digges (1605) explica que ela se refere à "linha c d", observe que, novamente, o autor apresenta os vértices da lateral do instrumento, utilizando letras minúsculas e a palavra "linha" para referir-se ao que hoje é conhecido, na escrita moderna, como segmento $(\overline{C D})$.

Ele ressalta que, para graduar a segunda escala do instrumento, ainda serão utilizados os valores da tabela de medidas da madeira. Contudo, observando com detalhes os valores da tabela, percebe-se que, a partir da $13^{\text {a }}$ linha, os valores estão em ordem decrescente e são referentes somente às polegadas e partes de polegadas, como pode-se verificar na Figura 5.

Observando a segunda escala do instrumento, constatou-se que ela não possui valores denotados, como na primeira escala. Nela, somente são feitas marcações referentes a cada uma das medidas. Com isso, conclui-se que os valores de 10 polegadas e 1/5 partes de polegadas, indicados pelas 13 polegadas quadradas, na tabela de medidas da madeira, informam a posição exata onde deve-se gravar a primeira medida da segunda escala, como pode-se ver na Figura 7.

\footnotetext{
${ }^{16}$ Em inglês, lê-se: "10 inchs, and 1/5. This must be numbered in the line $\mathrm{c}$ d from c which line betokeneth the thickness of the ruler" (DIGGES, 1605, XII, p.2).
} 


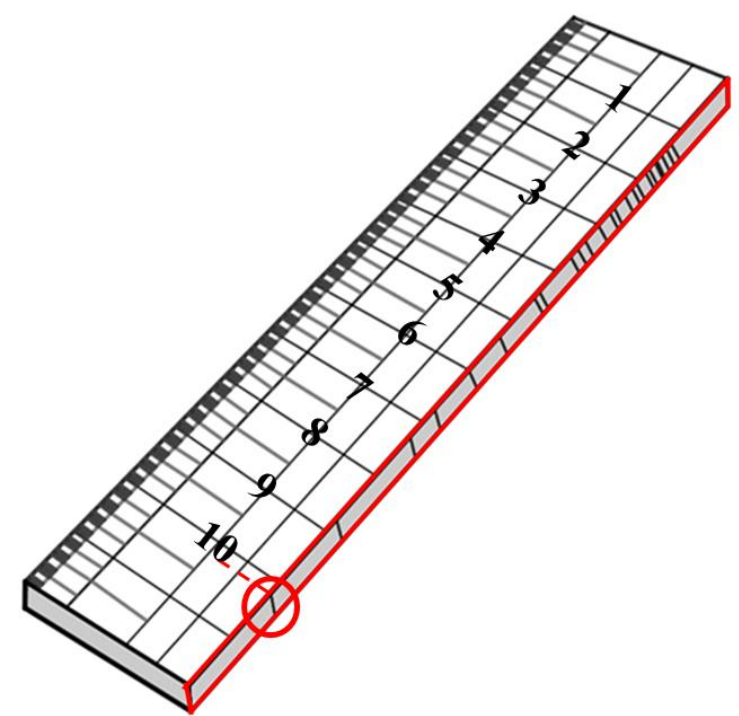

Figura 7: A primeira marcação da segunda escala (Elaboração das Autoras)

O mesmo processo é realizado para marcar as medidas referentes aos valores de 14 a 36 polegadas quadradas, representados na tabela de medidas da madeira, na segunda escala do instrumento, até que seja finalizada a sua graduação (Figura 8).

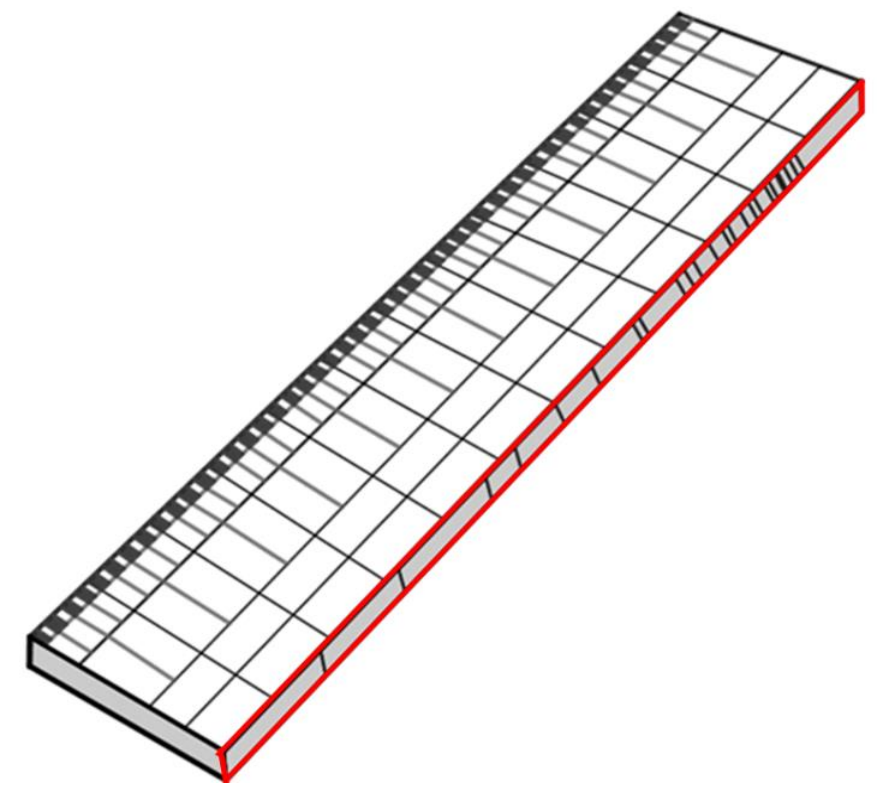

Figura 8: Segunda escala graduada da régua (Elaboração das Autoras)

Finalmente, a partir da realização desses processos, a parte da frente do instrumento está construída e graduada. Percebe-se que, para a realização dessa construção e graduação, não só o conceito de unidades de medidas é mobilizado, mas também conhecimentos a respeito de operações com frações. 


\section{Pensando matematicamente: a régua de carpinteiro no ensino de Matemática}

Observando com detalhes a régua de carpinteiro exposta no tratado de Digges (1605) e o processo realizado para graduar as escalas da primeira face, nota-se uma possível incorporação ao ensino, associada à articulação entre história e ensino de Matemática. De acordo com os Parâmetros Curriculares Nacionais (BRASIL, 1998), a História da Matemática pode apresentar aos alunos uma Matemática que é fruto de necessidades e preocupações humanas, que ocorreram em diferentes períodos da história e em sociedades e culturas distintas.

Analisando as informações apresentadas por Digges (1605), é possível identificar a mobilização de diversos conceitos matemáticos utilizados na Educação Básica e no Ensino Superior, relacionados, principalmente, à unidade temática de grandezas e medidas. Dentre esses conhecimentos, pode-se destacar a utilização de grandezas lineares (polegadas, pés, partes de polegadas).

Esse tema é abordado na Base Nacional Comum Curricular, de forma a “(EF03MA19) Estimar, medir e comparar comprimentos, utilizando unidades de medida não padronizadas e padronizadas mais usuais (metro, centímetro e milímetro) e diversos instrumentos de medida" (BRASIL, 2018, p. 289). Com isso, uma das formas de aplicação do instrumento régua de carpinteiro é que os alunos confeccionem suas réguas, utilizando a sua própria polegada como unidade de medida.

Uma vez que os valores dispostos na frente da régua estão distribuídos em quatro unidades de medida, sendo elas partes de polegadas, polegadas, pés e polegadas quadradas e que o objetivo da manipulação da régua, exposto pelo autor, é de medir pequenas distâncias, observa-se que a incorporação do instrumento ao ensino de Matemática pode contribuir para a construção de conceitos como unidades de medida, conversão de unidades de medida e comparação de comprimentos.

É importante ressaltar que o professor pode optar por realizar, com seus alunos, a situação de uso descrita por Digges (1605), cuja parte da frente da régua é utilizada, essencialmente, para encontrar o comprimento de pedaços de madeiras:

Suponha um pedaço de madeira para ser derretido, cujo quadrado verdadeiro é de 7 polegadas: este quadrado você o aponta para a figura do 7 na linha. g. h. sob o qual a direita no lugar atribuído a medida da madeira, está escrito de 2 pés. 11 polegadas e 2/7. Todas as vezes que essa medida é encontrada no 
comprimento de sua madeira, tantos pés de madeira estão nesse pedaço (DIGGES, 1605, XIV, p. 1) ${ }^{17}$.

Vale salientar que, antes de realizar a manipulação do instrumento em sala de aula, cabe ao professor apresentar aos alunos o conceito de unidades de medida e, também, indispensável dispor aos alunos todas as informações necessárias a respeito da régua de carpinteiro e sobre as unidades de medida nela contidas.

Por fim, visando mobilizar alguns dos conceitos matemáticos abordados na régua de carpinteiro, sugere-se que a aplicação do instrumento voltado para a conversão de unidades de medida, referente à graduação da parte $1 \mathrm{~b}$ da primeira escala, seja realizada na formação inicial do professor de Matemática, em que é possível propor uma discussão a respeito da incorporação de instrumentos matemáticos à Educação Básica.

\section{Considerações finais}

Diante das instruções fornecidas por Digges (1605), foi possível observar que não só a régua de carpinteiro, como a tabela de medidas da madeira, possui, em seu processo de construção e graduação, ferramentas que podem incorporar a História da Matemática à Educação Básica, uma vez que são mobilizados conhecimentos matemáticos para se obter os valores expostos na tabela, relacionando, assim, recursos didáticos diferentes dos que são abordados em sala de aula.

Vale salientar que um próximo passo para esta pesquisa, que ainda está em andamento, é realizar a reconstrução do instrumento régua de carpinteiro, visando o processo de graduação de suas escalas e a sua aplicação prática, com o foco principalmente voltado para a formação inicial do professor de Matemática, como forma de compreender as relações existentes entre as unidades de medida mobilizadas em suas escalas.

Além disso, é importante ressaltar que outro passo, que pode ser realizado, é a elaboração de atividades incorporando o instrumento régua de carpinteiro e a tabela de medidas da madeira, para que, assim, a sua aplicação prática possa expor as suas

\footnotetext{
17 "Suppose a piece of timber to be molten, whose true square is 7 inches: this square appointee you to the figure of 7 in the line. g. h. under whom rightward in the place assigned to timber measure, is written 2 foot. 11 inches, and 2/7. As often as that measure is found in the length of your timber, so many foot of timber is in that piece" (DIGGES, 1605, XIV, p.1).
} 
potencialidades didáticas.

\section{Referências}

ALFONSO-GOLDFARB, Ana Maria. O que é História da Ciência. São Paulo: Brasiliense, 1994.

BRASIL, Ministério da Educação. Secretaria de Educação Básica. Base Nacional Comum Curricular do Ensino Médio. Brasília: MEC/SEB, 2018.

BRASIL. Ministério da Educação. Secretaria de Educação Fundamental. Parâmetros Curriculares Nacionais: Matemática. Brasília: MEC/SEF, 1998.

CASTILlO, Ana Rebeca Miranda. Um estudo sobre os conhecimentos matemáticos incorporados e mobilizados na construção e no uso do báculo (cross-staff) em A Boke Named Tectonicon de Leonard Digges. 2016. 121f. Tese (Doutorado em Educação Matemática) - Faculdade de Ciências Exatas e Tecnologiaas. Pontifícia Universidade Católica de São Paulo. São Paulo.

DIGGES, Leonard. A boke named Tectonicon. London: Felix Kyngston, 1605.

DIGGES, Leonard. A boke named Tectonicon. London: Iohn Daye, 1556.

GIL, Antônio Carlos. Como elaborar projetos de pesquisa. 6 ed. São Paulo: Atlas, 2008.

GILLIPSIE, Charles Coulston. Leonard Digges. Dictionary of Scientific Biography. New York: Charles Scribner's Sons, 1971, 3. v., p. 97-98.

KRIPKA, Rosana Maria Luvezute; SCHELLER, Morgana; BONOTTO, Danusa de Lara. Pesquisa documental: considerações sobre conceitos e características na Pesquisa Qualitativa. In: CONGRESSO IBERO-AMERICANO EM INVESTIGAÇÃO QUALITATIVA, 4, 2015, Aracaju. Anais do CIAIQ2015. Aracaju: Universidade Tiradentes, 2015, p. 243-247.

PAULINO, Sabrina de Sousa; ARGEMIRO FILHO, Carlos Ferreira; PEREIRA, Ana Carolina Costa. Alguns aspectos contextuais da régua e do esquadro de carpinteiro no tratado A Booke Named Tectonicon (1556) de Leonard Digges. Boletim Cearense de Educação e História da Matemática, Fortaleza, v. 7, n. 20, p. 170-180, jul. 2020.

SAITO, Fumikazu. História da Matemática e suas (re)construções contextuais. São Paulo: Livraria da Física/SBHMat, 2015. 